\title{
La dimensión política del cuidado
}

Political Dimension of Care

\author{
Ells Natalia Galeano Gasca' (iD 0000-0002-9951-2043 \\ 'Universidad Manuela Beltrán, Vicerrectoría de Investigaciones, Bogotá, Colombia. \\ investigaciones@umb.edu.co
}

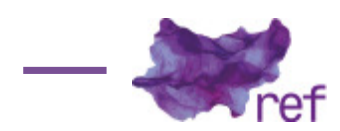

\begin{abstract}
ARANGO GAVIRIA, Luz Gabriela; AMAYA URQUIJO, Adira; PÉREZ BUSTOS, Tania; PINEDA DUQUE, Javier (Eds.).

Género y cuidado: teorías, escenarios y políticas.
\end{abstract}

Bogotá: Universidad Nacional de Colombia, Pontificia Universidad Javeriana, Universidad de los Andes, 2018.

El libro Género y cuidado: teorías, escenarios y políticas contiene reflexiones sobre la interrelación entre las categorías de género y cuidado desde diferentes perspectivas. La edición académica a cargo de Luz Gabriela Arango, Adira Amauya, Tania Pérez Bustos y Javier Pineda Duque resulta de un esfuerzo interinstitucional entre la Universidad Nacional de Colombia, la Universidad Javeriana y la Universidad de los Andes de Bogotá. Se abordan debates teóricos y aportes empíricos derivados de investigaciones de autoras/es adscritas/os a distintas instituciones alrededor del mundo, lo que permite vislumbrar cómo el fenómeno del cuidado mantiene ciertas continuidades en el nivel doméstico, local y global. Las aportaciones contribuyen a entender cómo la categoría de cuidado tiene un potencial político de importancia, sobre todo en lo referente a la necesidad de encontrar formas de relación más justas y equitativas, desde los espacios micro sociológicos que afectan la vida cotidiana, hasta los macro sociales que afectan a los colectivos.

El libro se encuentra divido en tres secciones: "Ética y ethos del cuidado", "Escenarios y significados del trabajo del cuidado" y "Organización social del cuidado y política pública". El primer capítulo, de autoría de Joan Tronto, es titulado "Economía, ética y democracia: tres lenguajes en torno al cuidado". La autora hace una reflexión ética desde la óptica del cuidado, vinculando aspectos relativos a la democracia. Igualmente, reflexiona sobre las atribuciones inequitativas de responsabilidades de cuidado y las asocia con las desigualdades de poder, expresadas en la clase social, la raza, la etnicidad, la sexualidad, entre otras diferencias. Aquí, la autora tipifica diversas formas de exención de las responsabilidades. Su enfoque intenta evitar que, al considerar la dimensión ética, se dejen de lado las preocupaciones sociales y estructurales, intentando tener presentes las dimensiones morales y las asociadas a la economía del cuidado. En este sentido, considera que es importante enmarcar el cuidado de manera que nadie se entienda ni totalmente dependiente, ni totalmente autónomo.

El segundo capítulo, "Responsabilidad y categorización de los destinatarios del cuidado", de Patricia Paperman, se enfoca en problematizar las diversas categorizaciones de los destinatarios del cuidado, provenientes de los estudios de discapacidad, conducidos por las personas que viven con esta condición, que reivindican su conocimiento y experiencia sobre ella, y de una corriente crítica de activistas y filósofas feministas que sostienen una postura más moderada sobre la concepción constructivista del cuerpo, la cual tiende a ignorar o infravalorar las realidades del 
cuerpo y sus problemas. Paperman problematiza el hecho de que las tareas de cuidado asociadas con lo masculino, como la protección en el trabajo de la policía, no se perciben como actividades de cuidado, mientras que las que pertenecen al ámbito privado, se relacionan con lo femenino y se perciben como la totalidad del universo del cuidado. Finalmente, la autora plantea la urgencia de considerar el cuidado como una necesidad fundamental en razón del reconocimiento de la vulnerabilidad humana y su interrelación con la presencia de cuidado adecuado, que, de no existir, desencadenaría un mal moral asociado con la injusticia y el sufrimiento. Por tanto, lo sitúa como un problema político.

Por otro lado, en el capítulo siguiente, denominado "El ethos del cuidado en la producción de concomimiento: una aproximación desde la antropología feminista al campo científico", escrito por Tania Pérez Bustos, se problematiza el hecho de que los productores de ciencia y sobre todo los referentes principales de las profesiones, son hombres blancos, europeos y muertos; en cambio, el ámbito de las profesiones del cuidado se encuentra asociado a la feminidad. Considera que esta situación contribuye a que el cuidado sea entendido como un oficio precarizado y marginal.

El cuarto capítulo, de Donny Meertens - "Re-tejiendo la sociedad campesina: género, cuidado y justicia en el posconflicto" -, gira en torno al papel de la mujer campesina en el posconflicto. Se basa en algunas de las reflexiones surgidas a lo largo de varias investigaciones en las que se evidenció el despojo y las resistencias campesinas, la búsqueda de justicia y restitución de tierras. Meertens plantea que la restitución implica un proceso de democratización de la sociedad en el que se transformen las condiciones de exclusión y de violencia estructural previa, devolviendo la dignidad y la autonomía. En este sentido, entiende a las mujeres como cuidadoras de personas, de tierras y animales en la economía familiar campesina y como reparadoras del lazo social dentro de comunidades rurales. Finalmente, plantea que el proceso de restitución formal e individual no actúa directamente sobre el reconocimiento, la autonomía o la dignificación del trabajo de cuidado de la tierra, que es realizado por las mujeres que viven el posconflicto.

En el capítulo cinco, de Pascale Molinier, "El 'trabajo sucio' y la ética del cuidado: historia de un mal-entendido", se entiende el cuidado desde el punto de vista del bienestar, tanto de las trabajadoras del cuidado como de aquellos a quienes cuidan por ser dependientes o enfermos. La autora argumenta que el bienestar de unas personas no puede sostenerse sobre la servidumbre de otras. De este modo, entiende al "trabajo sucio" como una serie de actividades complejas y consideradas repugnantes: lo que no se quiere hacer y, si es posible, se delega a alguien inferior en la jerarquía ocupacional.

En "Economías sexuales y percepciones sobre el cuidado", Adriana Piscitelli entiende el trabajo sexual como un servicio remunerado que se sitúa en el ámbito de intercambios económicos, sexuales y afectivos heterosexuales, los cuales tienen lugar fuera del casamiento. Allí es posible analizar la dimensión del trabajo emocional. Piscitelli realiza una tipología de las diferentes formas de prostitución posibles entre jóvenes brasileñas en distintos contextos. A través de esta, da cuenta de cómo se interrelaciona el afecto con las situaciones de dominación.

Por su vez, en el séptimo capítulo - "Servicios de cuidado y prácticas de reparación frente al racismo: salones de belleza para mujeres negras en Brasil" -, Luz Gabriela Arango hace referencia a los servicios estéticos y corporales, específicamente los servicios de peluquería, que pueden inscribirse en el campo del cuidado, en la medida en que se ocupan de las personas, su cuerpo, bienestar, dignidad social y autoestima. Señala que los servicios de cuidado estético y corporal están atravesados por contradicciones ligadas al mercado, a las desigualdades de clase, género, raza o sexualidad y al sentido que adquiere la apariencia en las sociedades modernas. Se relaciona, en primer lugar, con los efectos ambiguos de la belleza, entendida como una forma de opresión y alienación de las mujeres y, en segundo lugar, con el poder femenino subordinado. En su investigación se evidencia cómo el estilo del cabello resulta ser un vehículo para la expresión de la identidad que revela aspectos sociales y políticos.

Helena Hirata en el capítulo "Centralidad del trabajo del cuidado de las mujeres y nueva división sexual e internacional del trabajo" plantea que el trabajo del cuidado es el lugar donde se reflejan claramente las desigualdades imbricadas en el género, la clase y la raza, evidenciando que las cuidadoras son mayoritariamente mujeres, pobres, negras y muchas veces inmigrantes. Hirata realiza una investigación comparativa, tomando en cuenta las profesiones del cuidado en países tan diferentes como Brasil, Francia o Japón. Ella cuenta como la mercantilización y la externalización de dicho trabajo provocaron el envejecimiento de la población, por una parte, y la inserción masiva de las mujeres en el mercado laboral. En los tres países se trata de una profesión poco valorizada, con salarios relativamente bajos y escaso reconocimiento social.

El capítulo nueve se titula "El cuidado de los hijos e hijas de migrantes en el país de salida: construcciones de sentido y valoraciones" y fue escrito por Amparo Micolta León. Ella busca dar una respuesta al interrogante: ¿por qué madres, padres y abuelas asumen el cuidado de los hijos de mujeres y hombres migrantes? El trabajo de investigación que da origen a este texto muestra 
que las justificaciones que dan madres, padres y abuelas para cuidar los hijos de los y las migrantes están permeadas por discursos sustentados sobre la maternidad. A esto se suman distinciones asociadas a la condición conyugal y parental.

El capítulo "Repensar la organización social y política del cuidado infantil: el caso argentino", de Eleonor Faur, gira en torno a las políticas que intervienen en el cuidado de niños y niñas en Argentina. La autora plantea que se trata de un tramado de herencias y de enfoques constituidos a lo largo de las décadas, y que se cristaliza en la asignación desigual de derechos y prestaciones entre hombres y mujeres de distinta inscripción socioeconómica. De tal heterogeneidad, Faur busca entender cómo surge la configuración entre instituciones que regulan y proveen servicios de cuidado, y los modos en que los hogares de distintos niveles socioeconómicos acceden o no a ellos.

El capítulo once, "Entre el asistencialismo y los derechos de las mujeres: un análisis de la justicia de género del programa Más Familias en Acción en Colombia" fue elaborado por Yolanda Puyana Villamizar. Se refiere a la doble consecuencia del programa del Estado colombiano y que otorga a las mujeres un auxilio monetario, generando un etiquetamiento hacia la condición de pobreza de las mujeres, como también evidencia la manera en la cual la participación en el programa propicia espacios de empoderamiento colectivo.

Posteriormente, en el capítulo "La organización social del cuidado: políticas, desafíos y tensiones", Karina Batthyány conceptualiza el cuidado como la acción de ayudar a un niño, niña o persona dependiente en el desarrollo y el bienestar de su vida cotidiana. Ella propone un enfoque novedoso para entender el cuidado como un derecho, ya que es necesario recibirlo en distintas circunstancias y momentos del ciclo vital. Pero, al mismo tiempo, este enfoque implica que la satisfacción de esa necesidad no se determine por la lógica del mercado, la disponibilidad de ingresos, la presencia de redes vinculantes o lazos afectivos, sino que se establezca como un derecho universal al que todos debemos acceder; esto se complementa con el derecho a elegir si se desea o no tomarlo como una obligación. En cuanto a las políticas públicas en América Latina, la autora considera que se ha avanzado con lentitud, ya que el cuidado de las/os hijas/os y otros miembros de la familia, como los enfermos y las personas de edad, sigue siendo una actividad que recae desproporcionadamente sobre las mujeres. Finalmente, esto se relaciona con la falta de igualdad y la distribución desequilibrada entre trabajo remunerado y no remunerado.

En el capítulo siguiente, "Cuidado institucionalizado y vejez", Javier Pineda Duque busca analizar el trabajo de las cuidadoras de la vejez. Él argumenta cómo el proceso de la comercialización del cuidado de la vejez contribuye a su desvalorización, siendo que su institucionalización se torna insostenible. Además, plantea que el enorme costo y la cobertura limitada contribuyen a que los profesionales que trabajan en el cuidado sean mal pagados.

Mónica Toledo y Mirza Aguilar son las autoras del capítulo final titulado "Xocoyote: parentesco, género y cuidado no remunerado en el Altiplano Central mexicano". Ellas analizan el sistema familiar mesoamericano, a través del cual se prescribe la permanencia del ultimogénito en el hogar paterno para el cuidado de los padres; en contraprestación, este hereda la casa y las tierras. Si bien, este sistema recae en la figura masculina del xocoyote, es la esposa de este en quien recae directamente la responsabilidad del cuidado, evidenciándose la inequidad de género y la feminización del cuidado.

Esta variedad de contribuciones presentes en Género y cuidado: teorías, escenarios y políticas permite acceder al universo del cuidado desde discusiones teóricas y metodológicas que suponen una construcción de la categoría como un instrumento político. Adquiriendo una especial relevancia para problematizar las inequidades de género, sin desatender los aspectos relativos a la clase, raza y etnicidad.

\section{Bibliografía}

ARANGO GAVIRIA, Luz Gabriela; AMAYA URQUIJO, Adira; PÉREZ BUSTOS, Tania; PINEDA DUQUE, Javier (Eds.). Género y cuidado: teorías, escenarios y políticas. Bogotá: Universidad Nacional de Colombia, Pontificia Universidad Javeriana, Universidad de los Andes, 2018.

Ells Natalia Galeano Gasca (nataliagaleanog@gmail.com) es maestra en Antropología Social CIESAS CDMX Doctora en Antropología CIESAS CDMX. Ha trabajado en los últimos años en Colombia como docente de la Universidad de Antioquia, la Universidad CES y ha sido Investigadora del Grupo de Investigación Ocupación Humana, en la Línea de Investigación Participación Social, de la Universidad Manuela Beltrán. Actualmente se desempeña como apoyo técnico en la Gerencia de Salud Pública de la Gobernación de Antioquia en el componente de género y etnias. 


\section{COMO CITAR ESSE ARTIGO DE ACORDO COM AS NORMAS DA REVISTA}

GALEANO GASCA, Ells Natalia. "La dimensión política del cuidado". Revista Estudos Feministas, Florianópolis, v. 27, n. 2, e58049, 2019.

\section{CONTRIBUIÇÃO DE AUTORIA}

Não se aplica

\section{FINANCIAMENTO}

Não se aplica

\section{CONSENTIMENTO DE USO DE IMAGEM}

Não se aplica

\section{APROVAÇÃO DE COMITÊ DE ÉTICA EM PESQUISA}

Não se aplica

\section{CONFLITO DE INTERESSES}

Não se aplica

\section{LICENÇA DE USO}

Este artigo está licenciado sob a Licença Creative Commons CC-BY Internacional. Com essa licença você pode compartilhar, adaptar, criar para qualquer fim, desde que atribua a autoria da obra.

\section{HISTÓRICO}

Recebido em: 04/07/2018

Aprovado em: 24/11/2018 